\title{
Enhanced care - bridging the chasm
}

\author{
Authors: Sanjay Krishnamoorthy, ${ }^{A}$ Ratan Gor ${ }^{A}$ and Callum Jennings ${ }^{A}$
}

\section{Introduction}

Medical admissions are increasing in complexity - one in three admitted in 2015/16 had five or more health conditions, compared with one in 10 between 2005/6. Concurrently, the number of patients aged 85 and over being admitted to acute medical beds has grown at a greater rate than any other age group over the past decade. 1 The acutely unwell patient who cannot be managed on a general ward but does not meet the criteria for requiring a high dependency unit (HDU) poses a difficult problem. Although the care required in these situations does not fit the criteria for admission to the intensive treatment unit (ITU) or HDU, patients cannot safely be managed in a general ward environment due to the frequency of observations or the requirement of therapies difficult to deliver in non-specialist settings, eg non-invasive ventilation (NIV). Furthermore, elderly patients with complex comorbidities, increasingly have treatment escalation plans (TEPs) that preclude escalation to HDUs.

\section{Materials and methods}

Data collected at West Middlesex University Hospital (WMUH) indicated certain illnesses (Box 1) were not managed in the most appropriate settings as per national guidelines. A six-bed enhanced care unit (ECU) at WMUH aimed to provide an intermediary facility with enhanced monitoring, 1:2 nurse to patient ratio and therapies including NIV and nasal high flow therapy.

The ECU was staffed by a dedicated consultant and registrar, who form part of the medical emergency team, working to identify suitable patients. Dedicated training was provided to nurses on the unit to achieve Leve I 1/1+ enhanced care competencies. Prospective data were collected to analyse the impact on patient care.

\section{Results}

Between November 2018 and February 2019 there were 102 admissions to the ECU. Sepsis and respiratory failure were the predominant reasons for admission (Fig 1). Thirty-five per cent of patients received respiratory support through NIV.

Most patients were admitted for 2 days or less; four needed escalation to HDU/ITU. Four patients died while inpatients in ECU. Thirty-day mortality was $17.6 \%, 70.6 \%$ of patients survived until 100 days after admission. Fifty-six per cent of patients were at their ceiling of care, and not for resuscitation.

Authors: ${ }^{A}$ Chelsea and Westminster Hospital NHS Foundation Trust, London, UK

\section{Box 1. Conditions requiring enhanced monitoring}

Acute hypercapnic respiratory failure (requiring non-invasive ventilation)

Acute hypercapnic respiratory failure (requiring continuous positive airway pressure / nasal high flow oxygen therapy)

\section{Diabetic ketoacidosis}

Hyperosmolar hyperglycaemic state

Stage 3 acute kidney injury (Acute Kidney Injury Network criteria) Hyperkalaemia ( $>6 \mathrm{mmol} / \mathrm{L})$

Hypercalcaemia ( $>3 \mathrm{mmol} / \mathrm{L})$

Severe hyponatraemia $(<120 \mathrm{mmol} / \mathrm{L})$

Reg flag sepsis

\section{Conclusions}

The low conversion of patients from ECU to HDU/ITU indicates patients were appropriately identified for ECU provision.

Patients requiring NIV were provided safe care in line with best practice guidelines. $^{2}$

A new area for escalation was available for patients with hyperacute illnesses, complex comorbidities or significant frailty with appropriate ceilings of care placed.

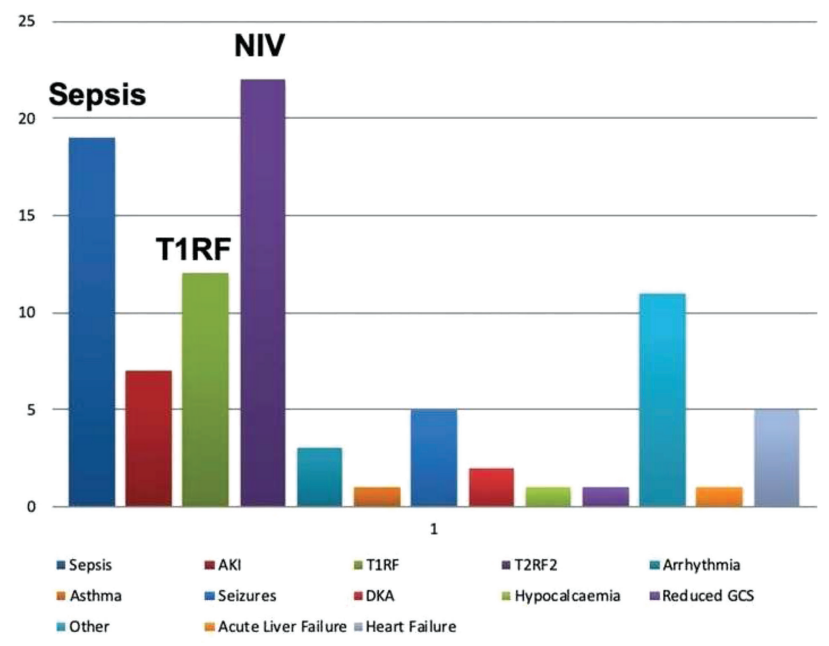

Fig 1. Breakdown of patients requiring enhanced care. 


\section{Next steps}

Following the success of the unit we have sought to establish a new level of care provision nationally. A national working group has been put together with representation from the three medical royal colleges, British Thoracic Society, Society for Acute Medicine and Faculty of Intensive Care Medicine to establish recommendations and standards for the delivery of enhanced care in medicine. This will include access to reproducible standard operating procedure documents and model business cases. We will seek to gain regulatory alignment with these standards to promote best practice.

\section{Conflicts of interest}

None declared.

\section{References}

1 Steventon A, Deeny S, Friebel Rocco, Gardner T, Thorlby R. Emergency Hospital admission in England: Which may be avoidable and how? London: The Health Foundation, 2018.

2 Davies M, Allen M, Bentley A et al. British Thoracic Society Quality Standards for acute non-invasive ventilation in adults. BMJ Open Respir Res 2018;5:e000283. 